\title{
Beneficial and limiting factors in return to work after primary total knee replacement: Patients' perspective
}

\author{
T.M.J. Pahlplatz ${ }^{\mathrm{a}, \mathrm{b}, *}$, M.U. Schafroth ${ }^{\mathrm{a}, \mathrm{b}}$, C. Krijger ${ }^{\mathrm{a}, \mathrm{b}}$, T.H. Hylkema ${ }^{\mathrm{c}}$, C.N. van Dijk ${ }^{\mathrm{a}, \mathrm{b}}$, \\ M.H.W. Frings-Dresen ${ }^{\mathrm{d}}$ and P.P.F.M. Kuijer ${ }^{\mathrm{d}}$ \\ ${ }^{a}$ Amsterdam UMC, University of Amsterdam, Department of Orthopaedic Surgery, Amsterdam, the Netherlands \\ ${ }^{\mathrm{b}}$ University of Amsterdam, Amsterdam Movement Sciences, Amsterdam, the Netherlands \\ ${ }^{\mathrm{c}}$ University Medical Center Groningen, Department of Orthopaedic Surgery and Department of Community and \\ Occupational Medicine, Groningen, the Netherlands \\ ${ }^{\mathrm{d}}$ Amsterdam UMC, University of Amsterdam, Department Public and Occupational Health, Amsterdam Public \\ Health research institute Amsterdam, Amsterdam Movement Sciences, the Netherlands
}

Received 13 September 2019

Accepted 1 September 2020

\begin{abstract}
.
BACKGROUND: Return to work (RTW) is an important outcome in Total Knee Arthroplasty (TKA). At present, 70-80\% of TKA patients return to work within three to six months.

OBJECTIVE: What are patients' perspectives regarding beneficial and limiting factors in RTW after TKA?

METHODS: Focus groups were formed in accordance with the Consolidated Criteria for Reporting Qualitative Research (COREQ) checklist. Three major topics were explored: 1 . What was beneficial for RTW after TKA; 2 . What was limiting for RTW after TKA; and 3. What additional care would benefit RTW after TKA?

RESULTS: Data saturation was reached after four focus groups, comprising 17 participants—nine men and eight women (median age 58, range 52-65). The focus group study identified four main themes that contributed to a successful RTW namely rehabilitation (medical) like post-operative physical therapy, patient characteristics (personal), like motivation to RTW, occupational characteristics (work-related) like build-up in work tasks and medical support (medical) like availability of a walker or crutches.

CONCLUSION: According to participants, factors within the following four themes can contribute to a successful return to work: occupational, patient, rehabilitation and medical care. Incorporating these factors into the integrated care pathway for the "young" TKA patients may increase the chances of a successful RTW.
\end{abstract}

Keywords: Total knee arthroplasty (TKA), return to work (RTW), employment, patient preferences

\footnotetext{
*Address for correspondence: T.M.J. Pahlplatz, Amsterdam UMC -Academic Medical Center location, Department of Orthopaedic Surgery, Meibergdreef 9, 1105AZ Amsterdam, The Netherlands. E-mail: t.m.pahlplatz@amsterdamumc.nl.
}

\section{Introduction}

In recent years, increased interest has been shown in the return to work (RTW) after Total Knee Arthroplasty (TKA). TKA is one of the most commonly performed orthopedic surgeries, with a mean age 
of 70 years. Due to the rising incidence of knee osteoarthritis, the number of patients receiving TKA globally is rising [1-3].

As a result, the number of younger patients undergoing TKA is increasing, leading to a higher proportion of patients returning to work after TKA $[4,5]$. Much research has been done regarding RTW rates and times after TKA, with studies conducted in several countries yielding similar results. A systematic review by Tilbury et al. showed that $71-83 \%$ of TKA patients returned to work, with an average time of 8-12 weeks [6]. Therefore, despite the success of the treatment, a significant proportion of patients are still not returning to work successfully. There is also significant variation in the time RTW can take, from $<1$ month to $>6$ months $[7,8]$.

A systematic review identified beneficial and limiting factors influencing RTW after TKA and described which patients were at risk of a prolonged rehabilitation period [9]. Patients that are male, have a high sense of urgency to return to work, have a high job qualification, are self-employed, with limited sick leave preoperatively are those that are most likely for a successful RTW. The included studies in the review also showed conflicting results regarding patient and work-related factors, both having positive or negative effects on RTW. To better understand these factors, the review emphasizes the need for more qualitative research $[9,10]$. The patient perspective must be taken into account to fully understand who is at risk of a prolonged or problematic RTW process, and when.

Five qualitative studies with a limited number of participants addressed this patient's perspective. Of those five studies only two studies, including a total of 18 patients, solely focused on patients that still have to work after TKA. Bardgett et al. concluded that delayed surgical intervention, limited advice regarding RTW, and a lack of work-focused rehabilitation contributed to a delay in successful RTW, in their study of 10 patients in England [10]. Mahdi et al. stated that unfulfilled patients expectations resulted in discontentment after TKA [11]. The personal situation of the individual patient, like the ability to work, is an important aspect in the patient's perspective stated by Barlow et al. [12]. A study by Gautreau concluded that patients experienced the rehabilitation period as a period of uncertainty which could be better addressed to enhance the outcome after TKA. [13] Following their study of eight patients in Canada, Maillette et al. stated when patients experienced difficulty in returning to work after TKA a biopsychosocial approach, incorporating genetic, personality and cultural factors, should be considered because other factors outside the biomedical care can play an important role in the rehabilitation [14].

To deepen the understanding regarding the patient's perspective and the biopsychosocial factors surrounding RTW this focus group study tried to identify not only factors limiting RTW but also the factors that contributed to a successful RTW. Moreover, appreciating the patient's perspective might enable a more focused vocational rehabilitation, given the importance of personal beliefs and expectations among this patient group [15]. These factors might enable the treating specialists to more readily identify patients at risk of a delayed or problematic RTW process.

The research question of this study is: what are patients' perspectives regarding beneficial and limiting factors in RTW after TKA?

\section{Methods}

\subsection{Study design}

We organized a series of four focus groups to explore the beneficial and limiting factors surrounding RTW after TKA among working-age patients. Data were collected using the Consolidated Criteria for Reporting Qualitative Research (COREQ), a 32-item checklist for interviews and focus groups [16]. The study was performed in accordance with the Declaration of Helsinki. The ethics committee of the Amsterdam UMC confirmed that the study (W14_111\#14,17.0144) is subject to the Dutch Medical Research Involving Human Subjects Act.

The focus groups were arranged at two separate locations, a conference room at the orthopedic department of the Amsterdam UMC and a designated conference room at the Amphia hospital in the Netherlands. Each focus group comprised of four to six participants, a moderator (TP), an administrator (PK, SH), a secretary (CK) and lasted approximately 1.5-2 hours.

The focus groups were conducted in a semistructured manner around three main topics: What are beneficial factors for return to work after TKA? What are limiting factors for return to work after TKA? Which possible factors would benefit return to work after TKA? Prior to the focus groups, the occupation, time of surgery, and duration of return to work of the participants were noted. It was also recorded 
whether they wished to be informed at the end of the study. During the focus groups, discussions between the participants were stimulated by the moderator and administrator. Study end-point was when data saturation was reached. All focus groups were provided with food and beverages, and travel expenses were reimbursed.

\subsection{Study sample}

From November 2014 to April 2016, participants were recruited (TP) from orthopedic outpatient clinics of the Amsterdam UMC and the Amphia hospital. An invitation to take part in the study was sent by postal mail.

The following inclusion criteria were used:

- Patients were in employment prior to the surgery

- Patients were under the age of 65 years

- Patients had undergone TKA 2-24 months prior to the focus group

- Patients must have had the intention to return to work after surgery

\subsection{Data analysis}

All focus groups were voice-recorded, and the recordings meticulously transcribed (TP, CK). These transcriptions were then checked against the original recording by the authors (TP, CK). Beneficial and limiting factors, as well as recommendations, were categorized into medical, work-related, personal and social themes, based on the ICF model [17].

The categorization was double-checked by one of the authors. If there was any disparity, a third author (PK) was consulted until consensus was reached about the specific category. The quotations have been edited for length, and pseudonyms have been used for the participants.

\section{Results}

\subsection{Participant demographics}

In total, 17 participants-nine men and eight women-were recruited, with a median age of 58 years (52-65). The jobs of participants were coded using the International Standard Classification of Occupations (ISCO-08) system. All patients were working preoperatively and had the intention to return
Table 1

Job categories and baseline characteristics of participants at the time of focus groups

\begin{tabular}{lcc}
\hline Focus group participants & Median age* & Sex \\
\hline & $58(52-65)$ & ○8 ○ 9 \\
Occupation & N $^{* *}$ & \\
Elementary occupations & 1 & \\
Craft and related trades workers & 4 & \\
Services and sales workers & 1 & \\
Technicians and associate professionals & 1 & \\
Professionals & 5 & \\
Disabled & 1 & \\
Unemployed/early retirement & 4 & \\
\hline
\end{tabular}

*Age: range $\min -\max$ in years. ${ }^{* *}$ number of participants.

to work. Four participants were unemployed or were in early retirement at the time of the focus groups. One patient was disabled and received disability benefits due to knee complaints as result of the TKA (Table 1).

\section{Findings}

The focus group study identified four main themes that contributed to a successful RTW. These themes were identified as related to the ICF modalities of medical, work-related, personal and social themes. The four main themes were based around themes of rehabilitation (medical), patient characteristics (personal), occupational characteristics (work-related), and medical support (medical). In total, 18 rehabilitation-, 20 personal, 17 occupational-, and 12 medical support-related factors were mentioned that positively or negatively influenced RTW.

\subsection{Theme 1: Rehabilitation}

Preoperative, in-hospital and postoperative physical therapy was found to be beneficial for a successful return to work (Table 2). The coaching role adopted by the physical therapist was found helpful in the rehabilitation, described regarding 'pushing boundaries'. T04 'In the beginning, when I was confronted with a lot of pain, the physical therapist was the one who stimulated me to go on.'

Structured home-based exercises provided by the physical therapist, with a gentle build-up in exercise hours, were considered beneficial. However, when the physical therapy was felt to be too intense for the patient or was creating too much stress on the mental wellbeing during the session, it limited the 
Table 2

Rehabilitation factors influencing RTW after TKA

\begin{tabular}{lll}
\hline Beneficial & Limiting & Possible Improvement \\
\hline Preoperative physical therapy & Too much exercise with physical therapist & Rehabilitation Center \\
In-hospital physical therapy & Stressful events during rehabilitation & Physical therapy \\
Postoperative physical therapy & Only home-based rehabilitation & Starting moving as soon as possible \\
Coaching role of physical therapist & & Good preoperative condition \\
Structured home exercises & & Availability of a home trainer \\
Availability of a home trainer & & Mobility scooter \\
Gentle build-up in exercise hours & & \\
Pushing through boundaries & & \\
Sufficient rest during rehabilitation & & \\
\hline
\end{tabular}

Table 3

Patient factors influencing RTW after TKA

\begin{tabular}{|c|c|c|}
\hline Beneficial & Limiting & Possible improvement \\
\hline Motivation to RTW & $\begin{array}{l}\text { Lack of energy during first two } \\
\text { months/fatigue }\end{array}$ & Staying positive/setting goals \\
\hline Motivation to return to sport & $\begin{array}{l}\text { Expectations that were set } \\
\text { too high }\end{array}$ & $\begin{array}{l}\text { Taking your time to recover/ } \\
\text { building in sufficient rest moments }\end{array}$ \\
\hline $\begin{array}{l}\text { Motivation to return stronger } \\
\text { than preoperatively }\end{array}$ & $\begin{array}{l}\text { High level of energy needed } \\
\text { for rehabilitation }\end{array}$ & $\begin{array}{l}\text { Be disciplined in your } \\
\text { rehabilitation }\end{array}$ \\
\hline Fear of losing work & Doing more than you can handle & Make a schedule \\
\hline \multirow{6}{*}{ Challenging yourself } & Poor preoperative condition & Listen to one's body \\
\hline & Inability to listen to one's body & \\
\hline & More time needed for recovery after TKA & \\
\hline & Overtraining & \\
\hline & Changed balance/coordination & \\
\hline & Altered sensation around the knee & \\
\hline
\end{tabular}

rehabilitation. T05: 'The physical therapist wanted to try and bend my knee and stated that it would not be a problem, but the knee was so swollen that I was not convinced that this was the best option. He did it anyway, and after it happened, it was just a mess and really set me back in my rehabilitation.'

Participants indicated that the physical therapist plays an important role and can positively influence patient motivation throughout the rehabilitation process.

Also participants indicated that the possibility to start using and training their "new" knee in a controlled environment, especially in the winter time, with the use of a home trainer was beneficial in the rehabilitation. T11: 'Purchase a home trainer, so you can start with training the knee as soon as possible.'

\subsection{Theme 2: Patient-related characteristics}

Participants indicated that they need to challenge themselves when they want a successful RTW Motivation to return to work, or to a sport, the fear of losing work, and a desire to return stronger than prior to surgery were all found to be factors beneficial for
RTW. T16: 'I wanted to return to work as soon as possible. That is where I was used to be during the day, not sitting at home looking out of the window.'

On the other hand, participants indicated to be aware of the unexpected lack of energy during the first two months and acknowledged that rehabilitation costs a lot of energy. T03: 'Your energy level after the operation is like a glass of water that is half empty, it does not take much to empty completely over the day.'

When patient expectations were set too high, or when they did more than they could handle, RTW was limited T12: 'You must take your time, train for yourself, you really need to practice.' (Table 3 ).

\subsection{Theme 3: Occupational characteristics}

Patients that had a phased, steady build-up in work tasks and work hours found this to be beneficial for a successful RTW. Also, patients who were in control of which tasks they had to perform, or the time allocated to do these in, found this to be beneficial in their RTW. T12: 'As soon as I was able to drive my car, I returned to work. I just was there; I was able to do whatever I wanted. I could plan my own schedule; if I wanted 
to sit or to go for a short walk it was $O K, I$ was given the opportunity to structure my own RTW.'

The patients that immediately encountered high workloads, or those who had to do standing work or tasks below knee level, found this limiting for their RTW. T01: 'After three months, you are at the mercy of your employer because you told him that the rehabilitation would take up around this amount of time, but when you need more time to recover there is a lot of pressure from the employer, and you need to justify why.'

Lack of support from an employer or the feeling of being pressured to return to work was found to be limiting. T01: 'You are not at your best moment in life in this period. A little support and contact from your employer are really helpful and important.'

One patient reported that incorrect guidance provided by the Employee Insurance Agency was unhelpful for his return to work (Table 4).

\subsection{Theme 4: Medical support}

Participant indicated that the use of the provided crutches or a walker and the presence of home care providers were beneficial factors for a successful RTW. T14: 'I did not use my crutches for four weeks after my surgery, but one day we went to the Zoo and I brought them with me and because of that I could walk the whole day without any complaint.'

Overall, the participants found medical support lacking in terms of patient information for RTW and often work was not addressed by the orthopedic surgeon. The pre-operative information provided was more designed for the older retired patient and the personal situation and history of the patient was not taken in to account. T03: 'The orthopedic surgeon knew that this was my fourth operation, but just after the operation, I heard the rehabilitation would take much longer than normal. How is that possible?'

Participants indicated that the post-operative outpatient clinic visits were short and felt impersonal. T02: 'The conversation with the doctor is often too short. You are often seen as just another knee or hip.' (Table 5).

\section{Discussion}

This qualitative study identified four important themes, consisting of rehabilitation-, patient-, occupation- and medical support-based factors that influence the process of successfully returning to work after TKA. Patient- and occupation-based factors are two domains identified in this study that are not described by the two previous qualitative studies on this topic [10, 14]. Regarding patient-based factors, greater appreciation of these factors - such as a patient's beliefs or motivations-could enable better expectation management by the orthopedic surgeon, or coping strategy provision by other treating specialists, such as the physical therapist and occupational physician, throughout the return to work process. This is in line with the meta-analysis of Dixon et al., who, whilst not specifically looking at RTW, showed that psychosocial interventions have a significant effect on the outcome of patients with arthritis [18]. With regard to occupation-based factors, this study illustrates that from the patient's perspective, a successful return to work after TKA is heavily influenced by, and should therefore involve, the work setting to which the patient has to return. This finding is supported by other studies, detailing that work environment and compensation policies are also important issues in the return to work process [19]. Early identification of occupational requirements, such as physical workload and associated risk factors, may increase the chance of a successful return to work.

This study also shows that rehabilitation- and medical support-based factors can contribute to a successful RTW. The focus group study of Bardgett et al. also identified these as two important themes. They stated that adequate provision of available rehabilitation facilities, and provision of limited and inconsistent medical advice, are important factors, positively and negatively respectively, in the RTW population [10]. Overall, participants in the current study found medical support lacking in terms of the patient information supplied regarding RTW-the information provided being more appropriate for the older, retired patient. Bardgett et al. also emphasized the need for a more tailored approach for this specific patient group [10]. This underlines an important aspect that patient-centered goals must be more incorporated by the treating specialists. Client-centered practice is a widely used strategy by occupational therapists not only regarding RTW or in patients with musculoskeletal disorders [20]. Norberg et al. showed that a client-centered treatment enhances energy levels in patient with heart failure and D'Cruz et al. showed patient centered goals improved ADL in patients with traumatic brain injury $[21,22]$. The important aspects in these treatments is 
Table 4

Occupational factors influencing RTW after TKA

\begin{tabular}{lll}
\hline Beneficial & Limiting & Possible Improvement \\
\hline Build-up in work tasks & Time pressure to RTW & Improved understanding of employer \\
Build-up in work hours & Doubt of employer regarding RTW & Building up work hours \\
Self-control in performing work tasks & Lack of support from employer & Building up physical tasks \\
Self-control in time needed to RTW & Inadequate support from Employee & Neutral guidance by occupational \\
& Insurance Agency & health physician \\
Doing chores around the house & High workload at starting work & Information for employer \\
& Doing standing work at start & \\
& Job tasks below knee level & \\
\hline
\end{tabular}

Table 5

Medical support factors influencing RTW after TKA

\begin{tabular}{|c|c|c|}
\hline Beneficial & Limiting & Possible Improvement \\
\hline $\begin{array}{l}\text { The use of a walker or } \\
\text { crutches }\end{array}$ & $\begin{array}{l}\text { Lack of patient information } \\
\text { regarding RTW }\end{array}$ & $\begin{array}{l}\text { Improved preoperative information on } \\
\text { rehabilitation/expectation management } \\
\text { regarding RTW }\end{array}$ \\
\hline \multirow[t]{6}{*}{$\begin{array}{l}\text { Presence of home care } \\
\text { providers }\end{array}$} & $\begin{array}{l}\text { Lack of knowledge of patient's } \\
\text { medical history on } \\
\text { the part of the orthopedic surgeon }\end{array}$ & More contact with orthopedic surgeon \\
\hline & $\begin{array}{l}\text { Lack of knowledge on the } \\
\text { part of the occupational physician } \\
\text { regarding TKA treatment }\end{array}$ & $\begin{array}{l}\text { More personal guidance from } \\
\text { the hospital }\end{array}$ \\
\hline & & $\begin{array}{l}\text { Better guidance throughout the rehabilitation } \\
\text { period from orthopedic surgeon }\end{array}$ \\
\hline & & $\begin{array}{l}\text { Have the surgery in the summer because of } \\
\text { better weather conditions when beginning to move }\end{array}$ \\
\hline & & $\begin{array}{l}\text { Improved information regarding RTW } \\
\text { throughout the rehabilitation }\end{array}$ \\
\hline & & Focus groups after treatment \\
\hline
\end{tabular}

the participation of the patient in the goal setting and planning and treatment process [23]. Incorporating a client-centered approach by the treating specialists could result in a more tailored treatment program which could enhance satisfactory short- and long terms results. A nice example is the recent study on Goal Attainment Scaling among working age knee arthroplasty patients that shows that preoperatively formulated patient-centered activity goals resulted in an increased satisfaction for these workrelated activities until 12 months post-operatively [24]

Another patient comment from the current study was that the orthopedic surgeon did not take into account the patient's medical history when advising on rehabilitation. This is supported by the qualitative studies of Mahdi et al. which concluded that the personal situation of the individual patient was not taken into account [11]. Other concerns described that the occupational physician could be lacking in specific knowledge about TKA and the postoperative guidance and possible risk factors for this patient group. This supports the conclusion of Bardgett et al. that this specific patient group-those who may return to work-require a more work-directed approach in guiding them through the rehabilitation process. This work-directed approach could enhance patient's expectations and lead to less discontentment after TKA. This corresponds with the conclusion of Barlow et al that unfulfilled patient's expectations resulted in discontentment after TKA [12]. This also corresponds to the patients' suggestions that more physical therapy sessions and a specialized rehabilitation center could improve the motivational support received from the physical therapists. This is also supported by the qualitative studies of Maillette et al. and Webster et al., which conclude that the physical therapists play an important role in keeping the patient motivated throughout the rehabilitation [14, 25]. The prospective study of Sharma et al. concluded that psychosocial factors like motivation enhance the functional outcome after TKA [26], which also underlines the importance of this aspect when guiding this specific patient population back to work. 


\subsection{Clinical implications}

The immediate success of TKA surgery is often measured in purely clinical results [27]: range of motion, postoperative pain, wound infection rates, and blood loss. Furthermore, the current care pathway is designed around a 'short-stay' principle, whereby the patient must be mobilized and discharged from hospital as soon as possible [28]. In the specific patient population in this study, the success of TKA is determined not only by these clinical outcomes but also by a successful and timely return to work. Incorporating RTW as a key factor in a care pathway may result in a more accurate and personalized rehabilitation schedule; allowing for adjustments throughout the rehabilitation process is designed to minimize potential delays and problems when RTW is appropriate. Improved patient information and better expectation management by the treating specialists may also increase the coping ability of the patient and thereby increase the success of the treatment in achieving a timely RTW. The four themes identified in this study can help the treating specialists and patients beforehand to formulate personalized treatment goals and a matching rehabilitation planning, for instance using Goal Attainment Scaling.

It is also important not only to emphasize the negatives, or potential sources of change, but also to build upon the aspects of care that were already beneficial, like, for example, in-hospital physical therapy. In doing this, we will achieve a better understanding of how we can support young knee arthroplasty patients to return successfully to work, and incorporate these findings into care pathways that contribute both to short-term clinical outcomes and longer-term outcomes, such as timely and sustainable return to work. In doing so, we can provide superior treatment for patients who may not currently be receiving the additional care needed to accomplish these important patient-centered goals [29].

\subsection{Strengths and limitations}

This study has several limitations. The study was conducted in two Dutch hospitals, and therefore the question can be raised as to whether this information applies to patients outside the Netherlands. However, the postoperative treatment for TKA is similar in most Western countries. Of course, the availability of postoperative physical therapy is not always covered by health insurance policies-as is the case in the Netherlands-in other countries. There is also the possibility of selection bias and whether the patients included in this study are representative of the young TKA patient. Although all patients had the intention to return to work, not everybody was successful in this at the time of the study. Four participants were unemployed, and one patient was registered as disabled and received sickness benefits due to knee complaints. Including these patients in the study reduces the risk of selection bias.

A strength of this study is that data saturation was reached. Moreover, compared to the other two focus group studies on RTW after TKA, we included a relatively high number of participants (17). Additionally, the participants represented a variety of stages in the rehabilitation process, ranging from 2-24 months after TKA. This provided information from patients who were still undergoing their rehabilitation, and patients who were able to look back on the entire process after rehabilitation and RTW. This proved to stimulate discussion among patients, as is the goal of a focus group.

\section{Conclusion}

This study showed that, according to patients, four overarching themes could contribute to a successful return to work. These include rehabilitation-, patient-, occupational-, and medical support-based factors. Better understanding and incorporation of these themes and their underlying constituent factors in the current care pathway by the treating medical specialists, such as the orthopedic surgeon, occupational physician and physical therapist, may improve the number of patients who can return to work successfully, as well as reducing the time required to return to work.

\section{Conflict of interest}

The authors have no conflict of interest to report.

\section{References}

[1] Carr AJ, et al. Knee replacement. Lancet. 2012;379 (9823):1331-40.

[2] Otten R, van Roermund PM, Picavet HS. Trends in aantallen knie- en heupartroplastieken: de vraag naar knie- en heupprotheses blijft voorlopig toenemen. [Trends in the number of knee and hip arthroplasties: considerably more knee and 
hip prostheses due to osteoarthritis in 2030]. Ned Tijdschr Geneeskd. 2010;154:A1534.

[3] Kuijer PPF, Burdorf A. Prevention at work needed to curb the worldwide strong increase in knee replacement surgery for working-age osteoarthritis patients. Scandinavian Journal of Work, Environment \& Health.

[4] Kurtz SM, et al. Future young patient demand for primary and revision joint replacement: national projections from 2010 to 2030. Clin Orthop Relat Res. 2009;467(10): 2606-12.

[5] Klit J. Results of total joint arthroplasty and joint preserving surgery in younger patients evaluated by alternative outcome measures. Dan Med J. 2014;61(4):B4836.

[6] Tilbury C, et al. Return to work after total hip and knee arthroplasty: a systematic review. Rheumatology (Oxford). 2014;53(3):512-25.

[7] Kuijer PP, et al. Which patients do not return to work after total knee arthroplasty? Rheumatol Int, 2016.

[8] Kievit AJ, et al. Total Knee Arthroplasty and the Unforeseen Impact on Return to Work: A Cross-Sectional Multicenter Survey. J Arthroplasty. 2014.

[9] Pahlplatz TMJ, Schafroth MU, Kuijer PPFM. Patientrelated and work-related factors play an important role in return to work after total knee arthroplasty: a systematic review. Journal of ISAKOS: Joint Disorders \& Orthopaedic Sports Medicine, 2017. May 2017.

[10] Bardgett $M$, et al. Patient-reported factors influencing return to work after joint replacement. Occup Med (Lond). 2016;66(3):215-21.

[11] Mahdi A, et al. Patients' experiences of discontentment one year after total knee arthroplasty- a qualitative study. BMC Musculoskeletal Disorders. 2020;21(1):29.

[12] Barlow T, et al. The decision-making threshold and the factors that affect it: A qualitative study of patients' decision-making in knee replacement surgery. Musculoskeletal Care. 2018;16(1):3-12.

[13] Gautreau S, et al. In their own words: A short report of patients' experiences of recovering from total knee replacement. Musculoskeletal Care. 2017;15(3):249-52.

[14] Maillette P, Coutu MF, Gaudreault N. Workers' perspectives on return to work after total knee arthroplasty. Ann Phys Rehabil Med. 2017;60(5):299-305.

[15] Hoorntje A, et al. Not Physical Activity, but Patient Beliefs and Expectations are Associated With Return to Work After Total Knee Arthroplasty. J Arthroplasty, 2017.

[16] Tong A, Sainsbury P, Craig J. Consolidated criteria for reporting qualitative research (COREQ): a 32-item checklist for interviews and focus groups. Int J Qual Health Care. 2007;19(6):349-57.
[17] Martins AC. Using the International Classification of Functioning, Disability and Health (ICF) to address facilitators and barriers to participation at work. Work. 2015;50(4): 585-93.

[18] Dixon KE, et al. Psychological interventions for arthritis pain management in adults: a meta-analysis. Health Psychol. 2007;26(3):241-50.

[19] Coutu MF, et al. Representations: an important key to understanding workers' coping behaviors during rehabilitation and the return-to-work process. J Occup Rehabil. 2007;17(3):522-44.

[20] Castro EM, et al. Patient empowerment, patient participation and patient-centeredness in hospital care: A concept analysis based on a literature review. Patient Educ Couns. 2016;99(12):1923-39.

[21] Norberg EB, et al. A client-centred programme focusing energy conservation for people with heart failure. Scand $\mathrm{J}$ Occup Ther. 2017;24(6):455-67.

[22] D'Cruz K, Howie L, Lentin P. Client-centred practice: Perspectives of persons with a traumatic brain injury. Scand J Occup Ther. 2016;23(1):30-8.

[23] Maitra KK, Erway F. Perception of client-centered practice in occupational therapists and their clients. Am J Occup Ther. 2006;60(3):298-310.

[24] Hoorntje A, et al. Goal Attainment Scaling Rehabilitation Improves Satisfaction with Work Activities for Younger Working Patients After Knee Arthroplasty: Results from the Randomized Controlled ACTION Trial. JBJS. 2020;102(16):1445-53.

[25] Webster F, et al. Understanding why people do or do not engage in activities following total joint replacement: a longitudinal qualitative study. Osteoarthritis Cartilage. 2015;23(6):860-7.

[26] Sharma L, et al., Prognostic factors for functional outcome of total knee replacement: a prospective study. J Gerontol A Biol Sci Med Sci. 1996;51(4):M152-7.

[27] Lange T, et al. Outcome Assessment in Total Knee Arthroplasty: A Systematic Review and Critical Appraisal. J Arthroplasty. 2017;32(2):653-665.e1.

[28] Monsef JB, et al. The impact of blood management on length of stay after primary total knee arthroplasty. Open Orthop J. 2014;8:108-13.

[29] Davis AM, et al. The trajectory of recovery and the interrelationships of symptoms, activity and participation in the first year following total hip and knee replacement. Osteoarthritis Cartilage. 2011;19(12):1413-21. 